\title{
New Ultrasound Signs for Prediction of Early Placental Insufficiency
}

\section{Original Article}

\author{
Tamer H. Said ${ }^{1}$, Gihan Mohamed Shehata', Yasser ELKassar ${ }^{1}$ \\ ${ }^{1}$ Department of Obstetrics and Gynecology, Faculty of Medicine, Alexandria University \\ ${ }^{2}$ Department of Biomedical informatics and Medical Statistics, Medical Research Institute, \\ Alexandria University, Egypt
}

\begin{abstract}
Background: Chronic placental insufficiency is a common condition that affects fetal growth and wellbeing. The response of the fetus to such stress depends on time and degree of placental affection. The acute placental insufficiency rapidly affects the fetal movements, amount of liquor and might lead to fetal demise while the chronic placental insufficiency is more deciduous and affects fetal weight and develops fetal growth restriction more slowly. The prophylaxis against the development of chronic placental insufficiency is more effective to protect growth and avoid perinatal morbidities and moralities.

Aim: Evaluation of 2 new ultrasound signs (tent like placenta and turbid liquor) to predict early placental insufficiency

Materials and Methods: One hundred pregnant women with gestational age between 16 weeks till 28 weeks were included in the study. This cohort of women with singleton pregnancy was evaluated by detailed ultrasound scanning to document gestational age, amniotic fluid index, cerebroplacental ratio (CPR), exclusion of congenital anomalies and other routine ultrasound scanning items. This cohort was divided into two groups, group one was the group with placental tenting and/or turbid liquor while group two was the group with no evidence of placental tenting or turbid liquor.

Results: Documentation of all relevant demographic data was done. The follow up was done every two weeks in all cases unless patients complained of decreased or absent fetal movement, passage of liquor, passage of blood. Spontaneous labour was allowed in primigravidae and elective cesarean section in Cases with previous c-section at completed 38 weeks of gestation was done unless one or more of the following signs had appeared that indicated the need for emergency delivery which were : abnormal low CPR less than 0.76, severe oligohydramnios, biophysical profile equal to or less than 4/8, abnormal cardiotocogram and fetal distress. The duration from the beginning of the study and documentation of the new studied signs in the placenta and turbid liquor till the development of placental insufficiency was calculated in weeks. All complications or adverse events were documented in both groups.

Conclusions: Both new signs were associated with increased risk for development of chronic placental insufficiency and can be used as predictors for evaluation of all pregnant women in the second trimester.
\end{abstract}

Key Words: Oligohydramnios, placental insufficiency, turbid liquor

Received: 06 May 2021, Accepted: 10 May 2021

Corresponding Author: Tamer Said, Department of Obstetrics and Gynecology, Faculty of Medicine, Alexandria University, Egypt, Tel.: 01223310490, E-mail: tamersaid697@gmail.com

ISSN: 2090-7265, May 2021, Vol.11, No. 2

\section{INTRODUCTION}

Chronic placental insufficiency is a common serious and potentially dangerous condition with many perinatal morbidity and mortality. Reduction of blood flow may compromise fetuses which have pre-existing hypoxia. The features of reduced baseline variability, decelerations, absence of accelerations suggest hypoxia in the fetus ${ }^{[1]}$.

Since there is a little to be done when diagnosis is made. Many studies tried to predict the development and progression of the placental insufficiency using ultrasound signs notable the uterine artery diastolic notch, fetal weight, doppler study of middle cerebral artery, umbilical artery, and amniotic fluid volume changes ${ }^{[2]}$.

The dilemma of strict definition of fetal growth restriction (FGR) and small for gestational age (SGA) hasn't been solved as there is a considerable overlap between both definitions. Fetal growth restriction (FGR) generally refers to a fetus that has failed to reach its biological growth potential because of placental dysfunction ${ }^{[3]}$.

Among many suggested ultrasonographic signs of decreased placental perfusion is CPR (Cerebroplacental Ratio) that was described for the monitoring of growth-restricted fetuses . It is currently considered as an early sign of placental chronic insufficiency; hence it could discriminate between constitutionally small and growth-restricted fetuses ${ }^{[4]}$.

Many biochemical markers as first trimester (pregnancy-associated placental protein-A and second trimester (total b-hCG and alpha fetoprotein) serum biochemistry could be used. 
Screening for placental insufficiency especially in high-risk pregnancies led to many interventions to improve the perfusion hence ameliorate the impact of hypoperfusion on the growing fetus. Many trials to improve the outcome were done using low dose aspirin either from the start of the pregnancy or from week 10 of the gestational age. Anti-thrombolytic therapy was used including low molecular weight heparin with aspirin to prevent recurrence of early pre-eclampsia in patients with inherited thrombophilia ${ }^{[5]}$.

Also, many drugs were tried to improve the placental circulation including sildenafi $₫ 1$, nitroglycerine $\AA$, minidose aspirin and antithrombotic therapy ${ }^{[6,7]}$.

Recent advances in novel imaging methods provide the basis for stepwise multi-parametric testing that may deliver cost-effective screening within existing antenatal care systems ${ }^{[8]}$.

\section{AIM OF THE WORK}

Evaluation of 2 new ultrasound signs (tent like placenta and turbid liquor) to predict early placental insufficiency

\section{PATIENTS AND METHODS}

This study took place in October 2018 till May 2020. This study included one hundred cases of pregnant women came for antenatal care in a private center for high-risk pregnancy. Inclusion criteria: all pregnant women with gestational age between 16 weeks till 28 weeks, age from 18 to 40 years were included in the study. The exclusion criteria included cases with body mass index less than 19 and more than $35 \mathrm{~kg} / \mathrm{m} 2$, preterm rupture of membranes or passage of liquor, smokers, chronic medical disease(hypertension, diabetes mellitus and rheumatic diseases) related to risk of low birth weight, positive consanguinity, multiple pregnancy, and known fetal congenital anomalies. This cohort of women with singleton pregnancy were evaluated by detailed ultrasound scanning to document gestational age, amniotic fluid index, cerebroplacental ratio (CPR) and 3 and 4 dimensional USG for exclusion of congenital anomalies. This cohort was divided into two groups, group one was the group with placental tenting and/or turbid liquor while group two was the group with no evidence of placental tenting or turbid liquor.

The placental tenting is the shape of the fetal surface of the placenta projecting into the liquor giving the shape of a tent and this results from the decrease of the pressure of the amniotic fluid needed to keep the fetal side of the placenta stretched. Turbid liquor is cloudiness or haziness of a fluid caused by large numbers of individual particles like smoke in air Figure (1-4).

All women were subjected to detailed medical and obstetric history, routine examination, and ultrasound scanning. Follow up of all cases was done every two weeks unless any complaints emerged. In every visit a
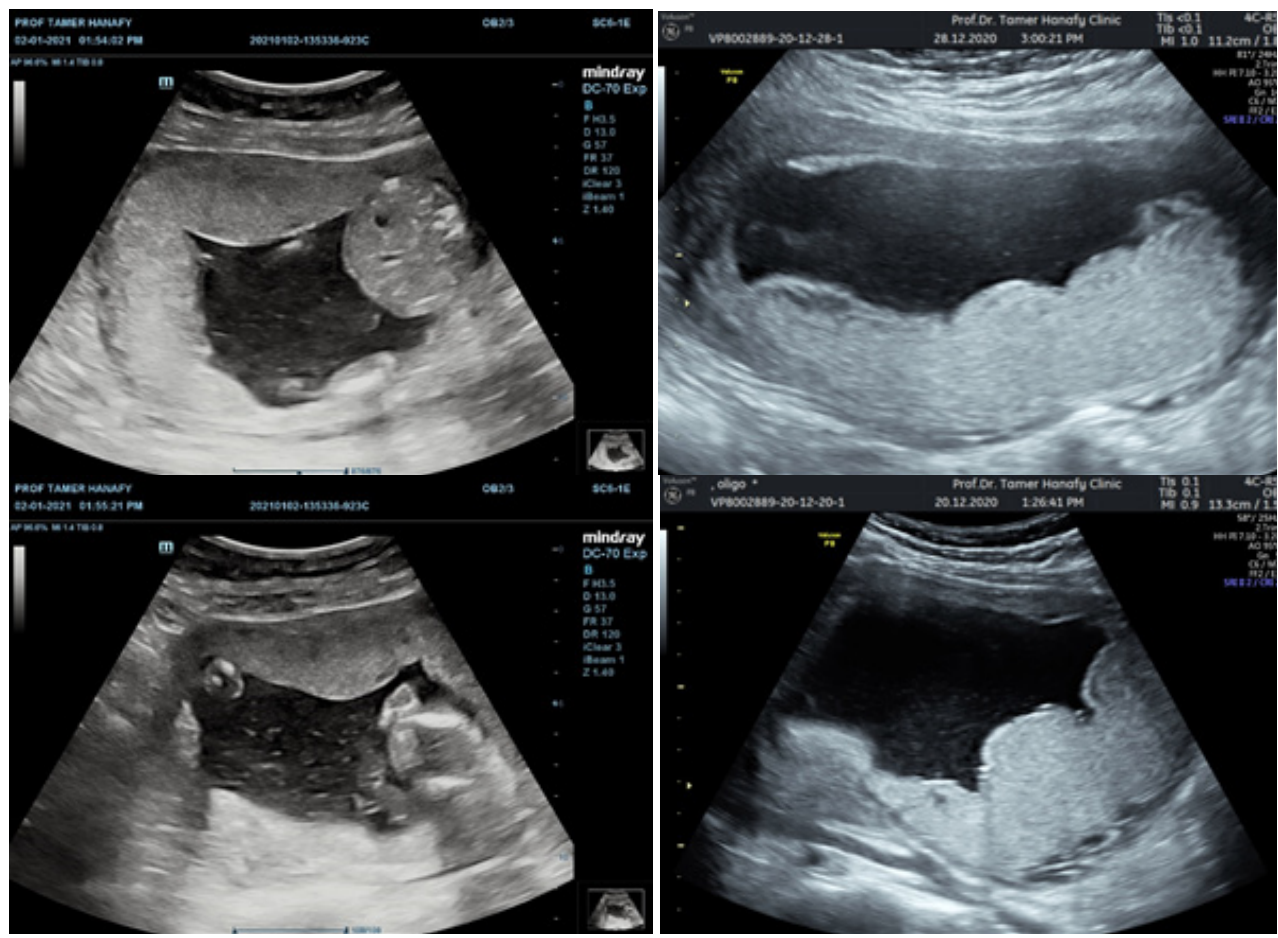

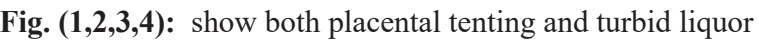


regular history taking, medical examination and obstetric ultrasound scanning waere done and measurement of AFI, CPR, fetal weight, biophysical profile, and presence of complications were documented ${ }^{[9]}$. All ultrasound scanning were done by the same sonographer (T.S.) using Voulson P8-version 2 general Electric company.

Documentation of all relevant demographic data was done. The bimonthly follow ups were done in all cases unless patient complained of decreased or absent fetal movement, passage of liquor, passage of blood in any of these previous situations the patient was asked to come and was evaluated immediately and managed accordingly. Spontaneous labour was allowed in primigravidae and elective cesarean section in previous c-section at completed 38 weeks of gestation unless one or more of the following signs appeared that indicated emergency delivery including abnormal low CPR less than 0.76, severe oligohydramnios, biophysical profile equal to or less than 4/8, abnormal cardiotocogram, and fetal distress.

The duration from the documentation of the new sonographic signs (placenta tenting and turbid liquor) till the development of placental insufficiency was calculated in weeks. All complications or adverse events were documented in both groups.

\section{STATISTICAL ANALYSIS}

Statistical analysis was performed using the IBM SPSS 20. The Shapiro-Wilk's test was performed to evaluate data distribution of all variables. Age, weight, parity, uterine size, and procedure time showed a normal distribution, and differences between groups were evaluated by two-tailed Student's t-test for independent data. Differences in pain score between the groups were calculated by T-student test. Statistical significance was set at $P<0.05$.

\section{RESULTS}

This study is a cohort observational one that aimed to evaluate 2 new ultrasound signs that could predict abnormal placental perfusion enough time before its exertion of its unfavorable effects. The demographic and clinical characteristics are demonstrated in (Table 1). The relative risk is $6.6(95 \% \mathrm{C}$. $\mathrm{I}=3.1-14.3)$. This means that those with placental tenting and or turbid liquor had 6 times risk to develop placental insufficiency. The distribution of the placental signs among both groups are shown in (Table 2).

There is agreement between the presence of placental tenting and or turbid liquor in $\mathrm{U} / \mathrm{S}$ and the presence of placental insufficiency (kappa statistic $=0.7, p$ value $=0.000)$ and that $87 \%$ of those with placental insufficiency has placental tenting and or turbid liquor compared to $13 \%$ of those without tenting or turbid liquor. This difference was statistically significant (chisquare test $=46.5, p$ value $=0.000$ ) and the risk estimate is shown in (Table 3). Complications in both groups are demonstrated in (Table 4). The complication rate was statistically significant higher in group (1) than in group (2).

Table 1: Comparison between group one and group two in demographic and clinical characteristics and pregnancy outcome

\begin{tabular}{|c|c|c|c|}
\hline & $\begin{array}{c}\text { Group one } \\
\text { Number } 50 \text { cases } \\
\text { Mean } \pm \text { standard deviation }\end{array}$ & $\begin{array}{c}\text { Group two } \\
\text { Number } 50 \text { cases } \\
\text { Mean } \pm \text { standard deviation }\end{array}$ & P value \\
\hline Age (years) & $29.6 \pm 5.5$ & $30.8 \pm 5.8$ & 0.30 \\
\hline Parity (median) & 0 & 1 & 0.25 \\
\hline Body Mass Index (kg/m2) & $26.7 \pm 4.3$ & $27.1 \pm 4.5$ & 0.63 \\
\hline Gestational age at recruitment (weeks) & $23.7 \pm 2.5$ & $24.2 \pm 2.6$ & 0.28 \\
\hline Amniotic fluid index at recruitment & $9.3 \pm 2.4$ & $14.4 \pm 2.4$ & $0.004^{*}$ \\
\hline Amniotic fluid index at delivery & $7.1 \pm 2$ & $11 \pm 1.8$ & 0.005 \\
\hline Cerebroplacental ratio at recruitment & $1.1 \pm 0.15$ & $1.1 \pm 0.14$ & 0.85 \\
\hline Cerebroplacental ratio at delivery & $1.2 \pm 0.4$ & $1.8 \pm 0.3$ & $0.006^{*}$ \\
\hline Duration till development of abnormal CPR & $9.8 \pm 1.6$ & $11.1 \pm 0.98$ & 0.06 \\
\hline Complications & $\begin{array}{c}34 / 50 \\
68 \%\end{array}$ & $\begin{array}{l}5 / 50 \\
10 \%\end{array}$ & $0.000^{*}$ \\
\hline
\end{tabular}

${ }^{*} P$ value is considered significant if less than 0.05 
Table 2: Placental tenting sign and or Turbid Liquor * Evidence of abnormal CPR Crosstabulation

\begin{tabular}{|c|c|c|c|c|}
\hline & & \multicolumn{2}{|c|}{ Evidence of abnormal CPR } & \multirow{2}{*}{ Total } \\
\hline & & Placental Insufficiency present & Placental Insufficiency absent & \\
\hline & Count & 40 & 10 & 50 \\
\hline \multirow[t]{3}{*}{ Group (1) } & Percentage & $80.0 \%$ & $20.0 \%$ & $100.0 \%$ \\
\hline & $\%$ within Evidence of abnormal CPR & $87.0 \%$ & $18.5 \%$ & $50.0 \%$ \\
\hline & Count & 6 & 44 & 50 \\
\hline \multirow[t]{4}{*}{ Group (2) } & Percentage & $12.0 \%$ & $88.0 \%$ & $100.0 \%$ \\
\hline & $\%$ within Evidence of abnormal CPR & $13.0 \%$ & $81.5 \%$ & $50.0 \%$ \\
\hline & Count & 46 & 54 & 100 \\
\hline & Percentage & $46.0 \%$ & $54.0 \%$ & $100.0 \%$ \\
\hline
\end{tabular}

Table 3: Risk Estimate

\begin{tabular}{|c|c|c|c|}
\hline & \multirow{2}{*}{ Value } & \multicolumn{2}{|c|}{$95 \%$ Confidence Interval } \\
\hline & & Lower & Upper \\
\hline Odds Ratio for Placental tenting sign and or Turbid Liquor & 29.333 & 9.774 & 88.031 \\
\hline For cohort Evidence of abnormal CPR = Placental insufficiency present & 6.667 & 3.108 & 14.302 \\
\hline For cohort Evidence of abnormal CPR = Placental Insufficiency absent & 0.227 & 0.129 & 0.399 \\
\hline $\mathrm{N}$ of Valid Cases & 100 & & \\
\hline
\end{tabular}

Table 4: Types and number of complications in both groups

\begin{tabular}{lccc}
\hline & Type of complication & Group (1) Number 50 cases & Group (2) Number 50 cases \\
\hline \multirow{3}{*}{ Complications } & Fetal Growth Restriction & 30 & 3 \\
& Intrauterine fetal death & 1 & 0 \\
No Complications & Severe Oligohydramnios & 1 & 0 \\
\hline
\end{tabular}

\section{DISCUSSION}

Placental insufficiency is a common finding that might lead to perinatal complications and morbidity. Once diagnosis of placental insufficiency is made limited choices are available to prevent the perinatal morbidity and the diagnosis is usually late. The aim of this study arises from the observation that many cases with borderline amniotic fluid index (intermediate oligohydramnios), that considered lower limit of normal, developed placental insufficiency few weeks later after that observation. This study aimed at evaluation of these ultrasound signs to predict early placental insufficiency and to save more time for early interference with supportive measures and improve perinatal outcome and hence decrease complications ${ }^{[10]}$.

Our study showed increased odds of development of early placental insufficiency if any of the two signs present. The presence of fetal hypoxia could increase incidence of oligohydramnios, growth restriction, antepartum fetal distress and stillbirth. Intrapartum complications are fetal hypoxia, acidosis, and higher rate of caesarean delivery. Neonatal complications include hypoglycemia, hyperbilirubinemia, meconium aspiration, persistent fetal circulation, hypoxic-ischemic encephalopathy, hypocalcemia, hyper-viscosity syndrome and necrotizing enterocolitis. Doppler assessment of the uterine, umbilical, middle cerebral vessels are used to identify placental insufficiency ${ }^{[11]}$.

Disruptions in early placental development, together with progressive pathological processes such as thrombosis, necrosis, and oxidative stress, combine to result into clinical syndromes that as a group are often termed 'placental insufficiency'. Pregnancies with these malfunctioned placentae usually complicated by severe pre-eclampsia, 'early onset' intrauterine growth restriction (IUGR), delivery of a small-for-gestational age infant before 34 weeks. Placental insufficiency usually diagnosed late and the prognosis from the time of diagnosis till delivery is not enough to reverse all the consequences resulted from damaged placenta ${ }^{[12]}$. In our study we observed about 8-11 weeks lapsed between diagnosis of any of proposed signs of prediction of early placental insufficiency and actual 
presence of placental insufficiency and this gave us a long time for interference and treatment.

It was suggested that careful evaluation of serum markers offers great promise as a screening tool to identify pregnancies at risk of serious placental insufficiency ${ }^{[13,14]}$. Many forms of severe placental insufficiency are described and characterized by small placentas with eccentric cords, termed 'chorionic regression', and a reduced maternal blood supply, termed decidual vasculopathy ${ }^{[15]}$. The placental pathology such as intervillous thrombosis (IVT) may be identified using real-time ultrasound and are termed echogenic cystic lesions ${ }^{[16]}$.

Many ultrasonographic measurements for diagnosis and evaluation of the placental perfusion function and to help in diagnosis of early placental insufficiency have been proposed include uterine artery resistance indices, AFI, umbilical and middle cerebral artery Doppler and CPR. The CPR is considered one of the important tools to diagnose and predict early onset chronic placental insufficiency. We used CPR between 1.0-1.1 as a reference curve ${ }^{[17]}$. or as a gold standard for diagnosis of impaired placental perfusion and we used CPR cut off at $0.6765 \mathrm{MoM}$ for interference and termination of pregnancy ${ }^{[18,19]}$.

Both AFI and single deepest pocket techniques for identifying true abnormal AF volumes. Both techniques were unreliable for identifying true $\mathrm{AF}$ volumes ${ }^{[20]}$. The signs described in this study are new, simple, and strongly correlated to increased risk for development of early placental insufficiency. This early prediction will give more time to investigate and interfere to prevent abnormal functioning placentae from causing perinatal morbidity and mortality. The increased rate of complications in group (one) was expected and anticipated and early interference prevented neonatal mortality except in one case. These results could be one of the simple answer for the need stated before about the deficiency of a solid screening or prediction tool for early placental insufficiency ${ }^{[21]}$.

Limitation of this study included limited number of patients, lack of neonatology reports of many patients as they delivered in different hospitals, and lack of laboratory results of few patients of inherited thrombophilia and rheumatic diseases.

\section{CONCLUSION}

Both new signs were associated with increased risk for development of chronic placental insufficiency and can be used as predictors of placental hypoperfusion and for evaluation of all pregnant women in the second trimester.

\section{RECOMMENDATION}

We recommend larger study and early work up to predict and diagnose the real onset and cause of development of early onset chronic placental insufficiency.

Consent for publication: Consent was obtained from all patients regarding participation and publication of the study results with all respect to confidentiality. All authors accept the publication.

\section{CONFLICT OF INTERESTS}

There are no conflict of interests.

\section{REFERENCES}

1. Jeph, R.B., et al., Serum Vascular Endothelial Growth Factor Levels in Normal Early Pregnancies And Recurrent Abortion Patients. Journal of Evolution of Medical and Dental Sciences,2018. 7: p. 5794

2. Figueras, F., et al., An integrated model with classification criteria to predict small-for-gestationalage fetuses at risk of adverse perinatal outcome. 2015. 45(3): p. 279-285.

3. Parra-Saavedra, M., et al., Association of Doppler parameters with placental signs of underperfusion in late-onset small-for-gestational-age pregnancies. 2014. 44(3): p. 330-337.

4. Gordijn, S., et al., Consensus definition of fetal growth restriction: a Delphi procedure. 2016. 48(3): p. 333-339.

5. Cruz-Martinez, R. and F. Figueras, The role of Doppler and placental screening. Best Practice \& Research Clinical Obstetrics \& Gynaecology, 2009. 23(6): p. 845-855.

6. Groom, K.M., et al., Enoxaparin for the prevention of preeclampsia and intrauterine growth restriction in women with a history: a randomized trial. 2017. 216(3): p. 296. e1-296. e14.

7. Coomarasamy, A., et al., Aspirin for prevention of preeclampsia in women with historical risk factors: a systematic review. 2003. 101(6): p. 1319-1332.

8. Audette, M.C. and J.C. Kingdom, Screening for fetal growth restriction and placental insufficiency. Seminars in Fetal and Neonatal Medicine, 2018. 23(2): p. 119-125.

9. DeVore, G.R.J.A.j.o.o. and gynecology, The importance of the cerebroplacental ratio in the evaluation of fetal well-being in SGA and AGA fetuses. 2015. 213(1): p. 5-15.

10. Toal, M., et al., Ultrasound detection of placental insufficiency in women with elevated second trimester serum alpha-fetoprotein or human chorionic gonadotropin. 2008. 30(3): p. 198-206. 
11. Erdem, M., et al., Comparative study of oxidative stress in maternal blood with that of cord blood and maternal milk. 2012. 285(2): p. 371-375.

12. Cross, J., et al., Genes, development and evolution of the placenta. 2003. 24(2-3): p. 123-130.

13. Rodesch, F., et al., Oxygen measurements in endometrial and trophoblastic tissues during early pregnancy. 1992. 80(2): p. 283-285.

14. Burton, G.J. and E. Jauniaux, Pathophysiology of placental-derived fetal growth restriction. American Journal of Obstetrics and Gynecology, 2018. 218(2): p. S745-S761.

15. Viero, S., et al., Prognostic value of placental ultrasound in pregnancies complicated by absent enddiastolic flow velocity in the umbilical arteries. 2004. 25(8-9): p. 735-741.

16. Chaddha, V., et al. Developmental biology of the placenta and the origins of placental insufficiency. in Seminars in Fetal and Neonatal Medicine. 2004 Elsevier.

17. Bahado-Singh, R.O., et al., The Doppler cerebroplacental ratio and perinatal outcome in intrauterine growth restriction. 1999. 180(3): p. 750756.

18. Kalafat, E. and A. Khalil, Clinical significance of cerebroplacental ratio. (1473-656X (Electronic)).

19. Morales-Roselló, J. and A.J.J.P.M. Khalil, Fetal cerebroplacental ratio and adverse perinatal outcome. 2016. 44(3): p. 355.

20. Magann, E.F., et al., Amniotic fluid index and single deepest pocket: weak indicators of abnormal amniotic volumes. 2000. 96(5): p. 737-740.

21. Stott, D., et al., Maternal demographics and hemodynamics for the prediction of fetal growth restriction at booking, in pregnancies at high risk for placental insufficiency. 2016. 95(3): p. 329-338. 